\title{
Doxazosin Effect on Neurogenic Bladder with Urine Retention in Female Dogs
}

\author{
Robert Cristian Purdoiu 1 ${ }^{*}$, Cristian Paul Popovici ${ }^{1}$, Răzvan Codea ${ }^{1}$, Mădălina Dragomir ${ }^{1}$, Caroline Lăcătuș ${ }^{1}$, \\ Laura Condor ${ }^{1}$, Mihai Musteață ${ }^{2}$, Sorin Marian Mârza ${ }^{1}$, Nicolae Coldea ${ }^{3}$, Radu Lăcătuș ${ }^{1}$
}

1 University of Agricultural Sciences and Veterinary Medicine Cluj Napoca, Faculty of Veterinary Medicine, Department of Clinical Sciences, 3-5 Manaștur, Cluj Napoca, Romania; robert.purdoiu@usamvcluj.ro, cristian.popovici@usamvcluj.ro, razvan.codea@usamvcluj.ro, madalina.dragomir@usamvcluj.ro, carolinelacatus@yahoo.com, condor.laura-ab@ansvsa.ro, sorin.marza@usamvcluj.ro, rlacatus2003@yahoo.com

2 University of Agricultural Sciences and Veterinary Medicine “Ion Ionescu de la Brad" Iași, Faculty of Veterinary Medicine, ; mihai.musteata@yahoo.com

3 "Dr Coldea Nicolea" private practice, 1C Tudor Arghezi St., Sibiu, Romania: coldeadvm@yahoo.com

* Correspondence: robert.purdoiu@usamvcluj.ro

Received: 23.04.2021

Accepted: 27.04 .2021

Published: 04.05.2021

DOI: 10.52331/cvj.v26i1.19

Copyright: (C) 2021 by the authors. Submitted for possible open access publication under the terms and conditions of the Creative Commons Attribution (CC BY) license

(http://creativecommons.org/licens es/by/4.0/).

\begin{abstract}
This short communication describes the effect of doxazosin in the case of urinary retention in female dogs due to motor neuron lesions produced by spinal trauma or spinal compression consecutively to intervertebral disk degeneration and extrusion. The study aims to determine whether the treatment of urinary retention in the case of neurogenic bladder in female dogs, using $\alpha_{1}$ adrenergic blocker, effectively promotes spontaneous recovery of the examined patients. Ten female dogs presenting with urinary retention were examined in the laboratory of Radiology, Faculty of Veterinary Medicine Cluj Napoca. The patients were examined using CT, radiography, and ultrasonography to identify the spinal cord injury responsible for the neurogenic bladder. The dose of doxazosin used in treating the voiding problem in the neurogenic bladder was $1 \mathrm{mg} / \mathrm{kg}$, a single dose per day. Depending on the spinal cord compression cause, the urine retention symptom was resolved.
\end{abstract}

Keywords: $\alpha 1$-adrenergic blocking agent, $\alpha$ adrenoreceptor, neurogenic bladder, upper motor neuron, dogs

\section{Introduction}

Spinal trauma and spinal compression are relatively frequent in dogs. Vertebral fractures and subluxation represent $7 \%$ of all neurological affection in dogs. Studies show that spinal trauma's most commonly affected location is T3-L3 representing 58\% [1]. The lumbosacral region is second-most affected by trauma representing $24 \%$ for the L4-L7 region and 7\% for S1-S3 region, more affected by the trauma of the sacral area are the small breed $13 \%[1,2]$.

The degenerative spinal compression is more prevalent in the chondrodystrophic breed, frequently affecting the T3-L3 spinal region [3]. From our experiences, the more frequent degenerative processes are encountered in the L7-S1 area in the larger breed.

The symptoms are related to the compression degree and may vary from mild manifestation to paralysis. Besides the locomotor system also the elimination of feces and urine is affected. The micturition process is regulated by circuits that involve parts of the autonomic and somatic nervous systems. Urine storage involves lumbosacral reflexes, and the spinal and encephalic centers control voiding of the urinary bladder. 
Spinal lesions located cranially of the sacral segment will determine an impairment of micturition and reorganization of the micturition reflex pathways. If the upper motor neuron is involved, an increase in urethral muscle tone will appear, determining the incapacity of voluntary emptying of the urinary bladder. Partial emptying of the urinary bladder can occur in a few days and is because of the presence of reflex pathways that involve the general visceral afferent, the general visceral efferent component of the pelvic nerve, and the general somatic efferent branch of the pudendal nerve, and activate the motor parasympathetic sacral neuron that inhibits the somatic neurons of the urethral muscle [4]. If the lower motor neuron is involved, the lesion is located at the level of the sacral plexus. Then the pelvic and pudendal nerves can neither hold nor evacuate urine, urine leaks, and the bladder is never distended $[4,5]$.

Electrophysiologic and histologic studies show that chronic injury of the spinal cord could induce phenotypic changes in bladder afferent neurons. Those changes include somal hypertrophy and increased expression of neurofilament protein, and increased excitability due to $\mathrm{Na}^{+}$and $\mathrm{K}^{+}$ion channels [5-7]. Other sympathetic mechanisms of the smooth muscle of bladder and urethra contraction involve the $\alpha_{1}$ and $\alpha_{2}$ adrenoreceptors that mediate the norepinephrine release by the postganglionic sympathetic terminals [5]. $\alpha_{1}$ adrenoreceptors suppress the effect based on a peripheral mechanism, while the $\alpha_{2}$ adrenoreceptors do so via a central mechanism [8-10]. Studies show that $\alpha_{1}$ adrenoreceptors exercise influence on the lower urinary tract function not only through the direct effect on the smooth muscle but also at the level of the spinal cord, ganglia, and nerve terminals and influence the outflow to the bladder, bladder, neck, prostate, and external urethral sphincter [11,12]. The influence of $\alpha$ adrenoreceptors vary depending on different condition. Studies show that the detrusor tissue from a patient with nonneurogenic bladder hyperactivity presents an increase in $\alpha$ adrenoreceptors compared to normal [12,13].

Multiples studies prove that $\alpha$ adrenoreceptors blocker plays an important role in treating acute urinary retention in case of benign prostatic hyperplasia (BPH) [12-16].

Management of neurogenic bladder in dogs that present spinal cord injury is a long-term problem. Actual management procedures consist of manual bladder voiding, periodic urinalysis, antibiotic treatment, and dietary/prophylactic antiseptic treatment $[17,18]$. Those procedures aim to address inappropriate urine reflex voiding that can result in urinary tract infection, increasing the risk of developing ascending pyelonephritis [19]. The use of doxazosin could prove a valuable addition in the early management of neurogenic urinary retention.

\section{Patients evaluation}

The effect of doxazosin was tested in ten female dogs that were presented for imaging evaluation of the spinal cord and present as one of the main symptom's urinary retention and bladder voiding problem.

The patients were clinically examined and were referred for imaging evaluation of the spine and spinal cord integrity. The clinical examination also follows the respiratory system and heart function without being identified any cardio-pulmonary pathologies. The respiratory, cardiac frequency and hematological and biochemical parameters were within normal limits. Four of the patients were diagnosed with spinal trauma located proximal to the sacral segment. Three of the patients presents fracture of the vertebral body, and one patient presented subluxation at the level of L5-L6. Three patients show degenerative pathology that produces mild to moderate compression of the spinal cord proximally to the sacral segment, and three patients were diagnosed with lumbosacral instability (LSI). 
The female group was heterogeneous, consisting of individuals of different breeds and ages. The common symptom was urine retention and bladder voiding problem. The other symptomatology related to the spinal cord injuries vary from mild to severe: lumbar pain manifested in palpation $(\mathrm{N}=4)$; proprioception deficiency on the hind limbs, minor walking deficit $(\mathrm{N}=3)$, paralysis of the rear limbs $(\mathrm{N}=3)$.

Other nonneurogenic causes that could produce urinary retention and dysuria, such as infections or urinary lithiasis, were ruled out using the medical imaging method.

Ultrasonographic examination of the urinary bladder and urethra show moderate to severe bladder distension in all patients, and contraction of the bladder neck or urethra was observed in four patients $(\mathrm{N}=4)$. In the other patients, the evaluation of the urethra through ultrasonography did not show abnormalities. Probing of the urethra was performed without any difficulties in the patients. The aspect of the urine was normal. When the bladder reaches full distention, the dogs can empty it, helped by abdominal massage.

The radiographic evaluation of the spine was relevant in the patients that presented spine trauma $(\mathrm{N}=4)$ and in the patients diagnosed with LSI $(\mathrm{N}=3)$.

The degenerative changes $(\mathrm{N}=3)$ and compression degree in case of trauma $(\mathrm{N}=4)$ were highlighted using Computed Tomography (CT). The CT scan shows the calcification of the intervertebral disks and narrowing of the intervertebral space $(\mathrm{N}=3)$. In the case of trauma patients, the spinal cord was evaluated, showing only compression without rupture of the soft tissue of the spinal cord.

Doxazosin is a postsynaptic $\alpha_{1}$-adrenergic blocking agent derivate from quinazoline used in managing hypertension, being useful in managing resistant hypertension as a component of combination therapy $[20,21]$. Doxazosin reduces the urinary symptom score and improves urinary flow in men with BPH $[11,12,22]$.

Alpha blocking agents were used in case of other pathology of the urogenital tract in humans to rebalance the cardiovascular system and restore blood volume and obtain peripheral circulation [23].

The dose of doxazosin used in treating urinary retention in the evaluated patient was $1 \mathrm{mg} / \mathrm{kg}$ administered in a single dose per day without using any other combination of alpha-blocker and promuscarinic medication.

\section{Discussion and conclusions}

The micturition process is mediated by sympathetic, parasympathetic, and somatic innervation. The mechanoreceptors give information concerning the urinary bladder distension in dogs in the urinary bladder wall [24]. Sensory information from the urethra, bladder wall, and bladder neck are transmitted via the lumbosacral dorsal root ganglia of the pelvic, hypogastric, and pudendal nerve to the spinal cord, in dogs the primary way for the impulse to travel is represented by the pelvic nerve [24,25]. In dog, the bladder filling and voiding process are regulated by the preganglionic nuclei of the sympathetic hypogastric nerves (facilitate filling of the bladder by relaxation and constriction of the urethra and bladder neck) and parasympathetic pelvic nerve (excite the detrusor muscle and void the bladder) located in the intermediate gray matter of L1-L3 and S1-S3 [19].

The parasympathetic micturition process relies on detrusor contraction due to postganglionic acetylcholine release binds with the muscarinic receptors in the urinary bladder wall. On the other 
hand, the sympathetic function is mediated by noradrenaline released from postganglionic neuron axon terminals that bind with $\beta$-adrenergic receptors within the bladder wall smooth muscle, producing detrusor relaxation, while binding with $\alpha 1$-adrenergic receptor within the urethral smooth muscle will result in urethral contraction [19,24].

"Spinal shock" that takes place in case of suprasacral spinal cord injury will produce bladder atony and urine retention due to post-injury impairment of the sympathetic and supraspinal micturition mechanism. The parasympathetic and somatic sacral innervation will remain involved in the voiding of the bladder, producing involuntary detrusor contraction during filling and uncoordinated sphincter contraction during voiding determines the high residual volume and high intravesical pressure $[19,26,27]$.

Depending on the administration route, doxazosin has high bioavailability in dogs. Oral administration bioavailability is $60 \%$. Absorbed doxazosin is subjected to complex biotransformation, and the metabolites are eliminated mainly in the feces. If administered orally, the percent of unchanged substance excreted in the urine in the dog is 3\% compared with $44 \%$ in feces. Intravenous administration determines a $12 \%$ entire substance be passed in the urine, and only $4 \%$ is excreted unchanged in the feces. The main metabolites result in the metabolism of doxazosin are common in human, mice, and dogs. The doxazosin presents moderate plasma clearance in dogs and a plasma elimination half-life value of $4.7 \mathrm{~h}$ [28].

In human medicine, $\alpha$-adrenoreceptors antagonist has become the main medication in patients with $\mathrm{BPH}$ and lower urinary tract symptoms (LUTS). Besides the sympathetic response that produces the smooth prostatic muscle relaxation, $\alpha$ blocker presents other mechanisms that can be involved in treating non-related BPH urine retention [12]. Different means of action include upregulation of $\alpha$ receptors in the bladder, $\alpha_{1 \mathrm{D}}$ receptors in the spinal cord, and dysfunction of the bladder neck and urethra [11]. Each of these could be influenced by pharmacological manipulation of $\alpha$ receptors [12].

Studies in males dogs suffering from vesicourethral reflex dysuria using $\alpha$-blocker show comparable results with those obtained in men. Treatment with $\alpha$-blocker shows an efficiency of $60 \%$ improvement of the urinary flow in dogs with vesicourethral reflex dysuria [29].

A study conducted on "prostate-like" symptoms in aging women shows no differences in women with LUTS. Compared the effect of doxazosin to hyoscyamine and anticholinergic, they show that $68 \%$ of the patients show improvement on monotherapy, and $77 \%$ had improvement on combined therapy. Also, 50\% of the patients that didn't respond to hyoscyamine show improvement to doxazosin [30]. The response being higher for doxazosin than for hyoscyamine [12,30]. Even if the effect of an $\alpha$-blocker is proven in women, there are few studies in the veterinary field that evaluate the impact of $\alpha$-blocker in female dogs [15].

In our cases, doxazosin administration in solitary dose shows improvement in urethral pressure and help to void the urinary bladder in all the patient. The rapid relief of the urine was present in L7-S1 compression when the symptoms improved after the first dose of doxazosin. In patients with degenerative disease and traumatic compression of the spinal cord, the symptoms improved after 2-3 administration of doxazosin. No side effect was registered after doxazosin administration. 
Doxazosin influences the adrenoreceptors of the urinary tract and produces relaxation of the smooth muscle in the detrusor and increases the bladder compliance, and affects the smooth muscle in the bladder and urethra neck by the use of $\alpha_{1}$-adrenoreceptors.

Doxazosin represents an effective medication for improving the symptoms in urinary retention due to neurologic bladder in female dogs. In the case of trauma-induced neurogenic bladder, the use of doxazosin help relieves urinary bladder pressure until surgical therapy is instituted.

Taking into consideration the complex pathology and low case number, the subject presents opportunities for further investigations.

Author Contributions: All authors have read and agreed to the published version of the manuscript".

Funding: Please add: "This research received no external funding."

Conflicts of Interest: “The authors declare no conflict of interest.”

\section{References}

1. Bali, M.S.; Lang, J.; Jaggy, A.; Spreng, D.; Doherr, M.G.; Forterre, F. Comparative Study of Vertebral Fractures and Luxations in Dogs and Cats. Vet Comp Orthop Traumatol 2009, 1, 47-53, doi:10.3415/VCOT-08.

2. Bagley, R. Spinal Fracture or Luxation. Veterinary Clinics of North America: Small Animal Practice 2000, 30, 133-153.

3. Forterre, F.; Gorgas, D.; M, D.; Jaggy, A.; Lang, J.; Spreng, D. Incidence of Spinal Compressive Lesions in Chondrodystrophic Dogs with Abnormal Recovery after Hemilaminectomy for Treatment of Thoracolumbar Disc Disease: A Prospective Magnetic Resonance Imaging Study. Veterinary Surgery 2010, 39, 165-172, doi:10.1111/j.1532-950X.2009.00633.x.

4. $\quad$ Nishizawa, O.; Sugaya, K. Cat and Dog: Higher Center of Micturition. Neurourol Urodyn 1994, 13, 169-79.

5. Yoshimura, N. Bladder Afferent Pathway and Spinal Cord Injury : Possible Mechanisms Inducing Hyperreflexia of the Urinary Bladder. Progress in Neurobiology 1999, 57, 583-606.

6. Bennett, B.C.; Roppolo, J.R.; Kruse, M.N.; de Groat, W.C. Neural Control of Urethral Smooth and Striated Muscle Activity in Vivo: Role of Nitric Oxide. J. Urol. 1995, 153, 2004-2009.

7. Black, J.A.; Langworthy, K.; Hinson, A.W.; Dib-Haji, S.D.; Waxman, S.G. NGF Has Opposing Effects on Na+ Channel III and SNS Gene Expression in Spinal Sensory Neurons. NeuroReport 1997, 8, 2331-2335.

8. Ramage, A.G.; Wyllie, M.G. A Comparison of the Effects of Doxazosin and Terazosin on the Spontaneous Sympathetic Drive to the Bladder and Related Organs in Anaesthetized Cats. Eur.J. Pharmacol. 1995, 294, 645-650.

9. Sugaya, K.; Nishijima, S.; Miyazato, M.; Ashitomi, K.; Hatano, T.; Ogawa, Y. Effects of Intrathecal Injection of Tamsulosin and Naftopidil, Alpha-1A and -1D Adrenergic Receptor Antagonists, on Bladder Activity in Rats. Neurosci.Lett. 2002, 328, 74-76.

10. Michel, M.C.; Vrydag, W. A 1-, A 2- and B-Adrenoceptors in the Urinary Bladder, Urethra and Prostate. British Journal of Pharmacology 2006, 147, 88-119, doi:10.1038/sj.bjp.0706619.

11. Andersson, K.; Lepor, H.; Wyllie, M. Prostatic A1-Adenoreceptors and Uroselectivity. Prostate. 1997, 30, $205-215$.

12. Nitti, V.W. Is There a Role for Alpha-Blockers for the Treatment of Voiding Dysfunction Unrelated to Benign Prostatic Hyperplasia? Reviews in urology 2005, 7 Suppl 4, S49-55.

13. Restorick, J.; Mundy, A. The Density of Cholinergic and Alpha and Beta-Adrenergic Receptors in the Normal and HyperReflexic Human Detrusor. Br J Urol. 1989, 63, 32-5.

14. Jeong, M.S.; Lee, J.G. The Role of Spinal and Peripheral A1- and A2-Adrenoceptors on Bladder Activity Induced by Bladder Distension and Anaesthetized Rat. BJU Int. 2000, 85, 925-931. 
15. Fischer, J.R.; Cribb, A.E. Urethral Pressure Profile and Hemodynamic Effects of Phenoxybenzamine and Prazosin in NonSedated Male Beagle Dogs Résumé. 1. Fischer JR, Cribb AE. Urethral pressure profile and hemodynamic effects of phenoxybenzamine and prazosin in non-sedated male beagle dogs Résumé. 2003;(858):30-8. 2003, 67, 30-38.

16. Karadenİz, A.; Pİşkİn, İ.; Eşsİz, D.; Altintaş, L. Relaxation Responses of Trigonal Smooth Muscle from Rabbit by Alpha 1 Adrenoceptor Antagonists Alfuzosin, Doxazosin and Tamsulosin Urinary Bladder Dysfunction Secondary to Benign Prostate Hyperplasia ( BPH ) in Humans and Animals Is a Major Health Prob. Acta Veterinaria Brno 2008, 77, 81-88, doi:10.2754/avb200877010081.

17. Bubenik, L.J.; Hosgood, G.L.; Waldron, D.R.; Snow, L.A. Frequency of Urinary Tract Infection in Catheterized Dogs and Comparison of Bacterial Culture and Susceptibility Testing Results for Catheterized and Noncatheterized Dogs with Urinary Tract Infections. Journal of the American Veterinary Medical Association 2007, 231, 893-899, doi:10.2460/javma.231.6.893.

18. Olby, N.J.; Mackillop, E.; Moore, S.; Mun, K.R.; Grafinger, M.; Osborne, J.A.; Vaden, S.L. Prevalence of Urinary Tract Infection in Dogs a Fter Surgery for Thoracol Umbar I Ntervertebral Di Sc Extrusi On. Journal of veterinary internal medicine / American College of Veterinary Internal Medicine 2010, 1106-1111.

19. Hu, H.Z.; Granger, N.; Jeffery, N.D. Pathophysiology, Clinical Importance, and Management of Neurogenic Lower Urinary Tract Dysfunction Caused by Suprasacral Spinal Cord Injury. Journal of Veterinary Internal Medicine 2016, 30, 1575-1588, doi:10.1111/jvim.14557.

20. Young, R.; Brogden, R. Doxazosin: A Review of Its Pharmacodynamic and Pharmacokinetic Properties, and Therapeutic Efficacy in Mild or Moderate Hypertension. Drugs. 1988, 35, 525-41.

21. Khoury, A.; Kaplan, N. $\alpha$-Blocker Therapy of Hypertension. JAMA. 1991, 266, 394-8.

22. Yuan, J.; Liu, Y.; Yang, Z.; Qin, X.; Yang, K.; Mao, C. The Efficacy and Safety of Alpha-1 Blockers for Benign Prostatic Hyperplasia: An Overview of 15 Systematic Reviews. Current Medical Research and Opinion 2013, $29,279-87$.

23. Calin, A.M.; Grigore, A.C.; Voicu, D.C.; Schaas, M.C. Toxic-Septic Abortion and Its Severe Complications, Frequently Lethal. Revista de Chimie 2016, 67, 2618-2622.

24. Uemura, EE. Chapter 22. Autonomic nervous system. In Fundamentals of Canine Neuroanatomy and Neurophysiology; WileyBlackwell, 2015; pp. 383-410.

25. Samson, M.; Reddy, VK. Localization of the Sacral Parasym- Pathetic Nucleus in the Dog. Am J Vet Res 1982, $43,1833-1836$.

26. Smith, P.M.; Jeffery, N.D. Spinal Shock-Comparative Aspects and Clinical Relevance. Journal of Veterinary Internal Medicine 2005, 19, 788-793, doi:10.1111/j.1939-1676.2005.tb02766.x.

27. Taweel, W. Al; Seyam, R. Neurogenic Bladder in Spinal Cord Injury Patients. Research and Reports in Urology 2015, 7, 85-99, doi:10.2147/RRU.S29644.

28. Kaye, B.; Cussans, N.J.; Stopher, D.A.; Hospital, S.G. The Metabolism and Kinetics of Doxazosin in Man, Mouse, Rat and Dog. Br. J. clin. Pharmac. 1986, 21.

29. Haagsman, A.N.; Kummeling, A.; Moes, M.E.; Mesu, S.J.; Kirpensteijn, J. Comparison of Terazosin and Prazosin for Treatment of Vesico-Urethral Reflex Dyssynergia in Dogs. Veterinary Record 2013, 173, 41-41, doi:10.1136/vr.101326.

30. Serels, S.; Stein, M. Prospective Study Comparing Hyoscyamine, Doxazosin, and Combination Therapy for the Treatment of Urgency and Frequency in Women. Neurourol Urodyn 1998, 17, 31-6. 